\title{
IMPACT DE LA DOLLARISATION SUR LES ACTIVITÉS ÉCONOMIQUES EN VILLE DE BUTEMBO
}

Héritier Kambale Kapitene

\author{
Editions ICES | « Revue Congolaise de Gestion »
}

2013/1 Numéro 17 | pages 171 à 200

ISSN 1729-0228

ISBN 2910153754

Article disponible en ligne à l'adresse :

https://www.cairn.info/revue-congolaise-de-gestion-2013-1-page-171.htm

\section{Pour citer cet article :}

Héritier Kambale Kapitene, «Impact de la dollarisation sur les activités économiques en ville de Butembo », Revue Congolaise de Gestion 2013/1 (Numéro 17), p. 171-200.

DOI 10.3917/rcg.017.0171

Distribution électronique Cairn.info pour Editions ICES.

(C) Editions ICES. Tous droits réservés pour tous pays.

La reproduction ou représentation de cet article, notamment par photocopie, n'est autorisée que dans les limites des conditions générales d'utilisation du site ou, le cas échéant, des conditions générales de la licence souscrite par votre établissement. Toute autre reproduction ou représentation, en tout ou partie, sous quelque forme et de quelque manière que ce soit, est interdite sauf accord préalable et écrit de l'éditeur, en dehors des cas prévus par la législation en vigueur en France. Il est précisé que son stockage dans une base de données est également interdit. 


\section{IMPACT DE LA DOLLARISATION SUR LES ACTIVITES ECONOMIQUES EN VILLE DE BUTEMBO}

\section{HéritierKAMBALE KAPITENE}

Faculté des Sciences Economiques et de Gestion

Université Officielle de Ruwenzori

Butembo - République Démocratique du Congo - B.P. 560 E-mail : hrtkapitene@,gmail.com

\section{Résumé.}

Ce texte démontre l'effet de la dollarisation sur les activités économiques à Butembo. De 2007 à 2011, les prix ont été 4,86305 fois ceux du premier trimestre 2007 en augmentant de 386,3\% par rapport au premier trimestre 2007 et ont augmenté de 266,1\% par rapport au premier trimestre. Le taux de dollarisation affecte le taux de change en 87,73\%.Le taux d'inflation est expliqué en 50,57\% par celui de dollarisation. Les mécanismes de variations des prix des produits tiennent à un certain seuil sur l'usage de l'une ou de l'autre monnaie, entre le dollar américain et le franc congolais.

Mots Clés : Dollarisation, Taux de Change, inflation, Dedollarisation, Prix. 


\section{Abstract.}

This paper shows the effect of dollarization on economic activities in Butembo. From 2007 to 2011, prices were 4.86305 times that of the first quarter of 2007 increased $386.3 \%$ compared to the first quarter of 2007 and increased by $266.1 \%$ compared to the first quarter. The dollarization rate affects the exchange rate $87.73 \%$. The inflation rate is explained by the $50.57 \%$ of dollarization. Mechanisms of changes in commodity prices take a certain threshold on the use of one or another currency, the U.S. Dollar and the Congolese franc.

Keywords: Dollarization, Exchange Rate, inflation, Dedollarization,Price

\section{INTRODUCTION}

T a dollarisation de l'économie d'un pays est l'état où sa Lmonnaie nationale n'exerce plus les fonctions monétaires de réserve de valeur, d'unité de compte et d'intermédiaires aux échanges. Ces fonctions sont donc exercées par une (des) devise(s) étrangère(s) qui se substitue (nt) à la monnaie nationale. L'exercice des fonctions monétaires par une devise étrangère prouve un niveau de méfiance que la population porte à l'égard de la monnaie nationale. A la lumière de ce qui précède, l'incertitude que fait régner l'instabilité sociopolitique sur la valeur et sur la détention de la monnaie nationale et surtout sur l'avenir même du pays explique l'éclosion, le maintien, voire l'accentuation de la dollarisation de l'économie congolaise (Vahavi, [2007], p.17).

Dans la vie socioéconomique congolaise en général et celle de Butembo en particulier, une libre observation montre que le dollar américain est utilisé comme réserve de valeur, unité de compte et moyen de paiement. En fait, la plupart des dépôts bancaires, de transferts bancaires, les prix 
de produits bruts et manufacturés, les prix des services comme la location des maisons, du matériel ou du mobilier; le transport, l'entretien et réparation des biens meubles et immeubles; les soins de santé, les frais scolaires et académiques, etc.; presque tout s'effectue moyennant le dollar des Etats-Unis (dollar américain). Ainsi, le dollar constitue désormais la référence permanente et permet, de ce fait, de garantir l'unité de compte pour l'achat des biens et services marchands.

De ce fait, il convient de mentionner que la dollarisation a constitué une entorse à notre monnaie nationale dans ses différentes fonctions. Par conséquent, la dollarisation de l'économie congolaise serait, bien entendu, l'une des contraintes pour le déploiement d'une politique monétaire efficace (Sekeraviti, [2007-2008], p.35).La réflexion à mener face à ce problème chercherait à savoir si cette tendance, qu'ont les différents acteurs de l'économie congolaise, de se tourner vers le dollar américain n'a pas d'effets néfastes sur l'économie congolaise en général et particulièrement sur celle de Butembo. En effet, la quantification des effets d'une dollarisation est intrinsèquement difficile, des nombreux modèles l'ont tenté et ont autorisé des prévisions très diverses (Gaetano A. et Keister T., [2002], p.18).

Ainsi, dans notre étude nous voulons savoir si la dollarisation a un impact sur l'appréciation de la monnaie nationale et sur le niveau de prix en ville de Butembo. D'une manière anticipative, nous estimons que :

La dollarisation provoquerait une dépréciation de la monnaie nationale face au dollar américain sur le marché de Butembo.

Il se pourrait que cette dollarisation occasionne une hausse du niveau général de prix des produits sur le marché 
local, relation dans laquelle la variation du taux de change constituerait un effet de transition.

- Ce travail est motivé par la recherche ou l'identification des conséquences des changements dans l'appréciation des individus à l'égard de la monnaie congolaise. Ce travail a pour objectif de déterminer les conséquences de la substitution monétaire sur la valeur de la monnaie nationale, face au dollar des Etats-Unis, et sur le niveau de prix des produits en ville de Butembo. Ainsi donc, à l'issue de notre analyse, la présente étude apportera une idée concernant l'influence de la dollarisation sur la variation du taux de change du dollar - en francs congolais - et sur le taux d'inflation en Butembo. C'est là que s'impose son intérêt majeur du fait qu'elle saisit un fait nouveau - la dollarisation - dans l'explication des phénomènes économiques précités.

Nous usons de la méthode systémique qui nous permet de déceler les facteurs en liaison avec le phénomène et d'identifier les interactions de ces différents facteurs par rapport au phénomène en étude. Les approches analytique et économétrique nous aident à mener certaines analyses, à estimer des modèles économétriques et de construire des tests statistiques y relatifs. Ces approches et méthode sont soutenues par la technique documentaire, l'observation des faits et l'interview, tout en portant sur la ville de Butembo, pôle économique majeure au Nord Kivu à l'Est de la République Démocratique du Congo pour une période allant de 2007 à 2011.

\section{LA DOLLARISATION ET SES MECANISMES}

\subsection{Définition de la dollarisation}

Issu du phénomène de substitution monétaire, dans certains pays d'Amérique latine où le dollar des Etats-Unis s'est substitué aux monnaies nationales, le concept de 
dollarisation fait l'objet de plusieurs définitions. Melvin (1988) considère la dollarisation comme un phénomène de demande de monnaie étrangère par les résidents au lieu de la monnaie nationale. Il la définit ainsi comme l'utilisation des dollars américains par les résidents de l'Amérique latine à la place de la monnaie locale. Calvo et Vegh (1993), quant à eux, la considèrent comme le concept le plus approprié pour décrire le phénomène où la monnaie domestique est graduellement remplacée par la monnaie étrangère, généralement le dollar des Etats-Unis, d'abord dans sa fonction de réserve de valeur puis dans celle d'unité de compte et enfin de moyen de paiement. Dans un sens plus large, Bourguinat et Dohni (2000) soulignent que la dollarisation est le processus de remplacement, par le dollar des Etats-Unis, des monnaies nationales préexistantes en tant qu'unité de fixation du prix des biens, d'instrument des paiements et de détention de l'épargne.

Ce concept «dollarisation » présente une similitude à celui de «substitution monétaire ». Pour ce dernier concept, Brand (1994) part de l'hypothèse que dans un pays en développement, la perte par la monnaie nationale d'une ou de plusieurs de ses fonctions vaut substitution monétaire. Cet auteur donne une différence du sens du phénomène de substitution monétaire pour les pays industrialisés et les pays en développement. Il souligne que, pour les premiers, compte tenu de la forte intégration des marchés mondiaux et du développement important des marchés de capitaux, la substitution monétaire résulte essentiellement d'une diversification des portefeuilles de titres des investisseurs et de l'effort réalisé par les importateurs et exportateurs pour réduire leurs coûts de transaction dans le cadre du commerce international. Par contre, pour les seconds, qui dans la plupart connaissent une instabilité politicoéconomique et une inefficience ou quasi inexistence des 
marchés financiers, la substitution monétaire traduit essentiellement le fait que les agents économiques se détournent de la monnaie nationale.

C'est dans cette renonciation que les devises étrangères sont utilisées pour leur fonction de réserve de valeur, d'unité de compte et de moyen de paiement sur le territoire national. Nous comprenons par là que ces définitions intègrent le concept de remplacement pour élucider celui de substitution monétaire alors qu'on reconnaît qu'il existe, dans des pays, la cohabitation de deux ou plusieurs monnaies. Ce qui pose un problème du degré d'usage de la monnaie étrangère dans les pratiques monétaires nationales. Il y a donc ici une complémentarité entre monnaies en ce sens que la monnaie étrangère assume les fonctions que la monnaie nationale assume mal ou ne peut plus assumer. Ainsi donc, comprenons-nous que la substitution est un synonyme du concept dollarisation exprimant la défiance des agents économiques à l'égard de la monnaie nationale, un processus où la monnaie étrangère, le dollar des Etats-Unis, assume progressivement les fonctions d'unité de compte, de réserve de valeur et d'intermédiaire des échanges.

\subsection{Les régimes de dollarisation}

La dollarisation est un concept multiforme; la classification de ses régimes repose sur deux indicateurs : le degré de pénétration de la monnaie étrangère- le dollar américain- dans les pratiques monétaires du pays dollarisé et le degré d'officialisation de son usage par les autorités du pays. Tenant compte du degré de pénétration du dollar, on distingue la dollarisation partielle de la dollarisation intégrale ou totale. Se référant au degré d'officialisation, on distingue la dollarisation officielle ou de droit de la 
dollarisation officieuse ou non officielle ou encore de fait (Vahavi, [2007], p.76).

De ce fait, partant des différents critères évoqués cidessus, nous pouvons retenir les explications suivantes : La dollarisation partielle explique la situation dans laquelle la monnaie étrangère s'intègre au dispositif monétaire préexistant pour constituer une pluralité monétaire alors que la dollarisation intégrale s'observe lorsque la monnaie étrangère circule en tant que signe monétaire exclusif dans une économie sans monnaie propre.

On parle de dollarisation officielle lorsque l'usage de la monnaie étrangère dans l'économie est reconnu officiellement par les autorités et à travers la déclaration de son cours légal; elle relève donc d'un choix public tandis que la dollarisation officieuse survient lorsque la monnaie étrangère est utilisée par les agents économiques en dépit de l'absence de son cours légal; c'est donc un phénomène spontané relevant d'un choix des agents privés. Il sied ainsi de comprendre que les dollarisations officielle et officieuse peuvent être chacune soit partielle, soit intégrale.

\subsection{Les facteurs explicatifs de la dollarisation}

\subsubsection{La dollarisation par la crise inflationniste}

Lorsque l'inflation atteint un niveau élevé, les résidents s'obligent à détenir leur richesse financière sous forme de billets ou/et en monnaies étrangères. Aucun intérêt de détention de richesse sous forme d'une monnaie qui perd sa valeur au jour le jour ne se justifie plus. La monnaie étrangère se substitue à la monnaie interne en tant que réserve de valeur, par la détention manuelle ou dans des comptes bancaires domestiques. Cette constitution de l'épargne en monnaie étrangère traduit la perte, par la monnaie nationale, de sa fonction de réserve de valeur. 
Dès lors, les agents économiques commenceront à libeller non seulement les prix des biens durables et des immeubles en monnaie étrangère d'abord, puis ceux des biens courants, mais aussi les dettes et les contrats. Il se vérifie, ici, la perte par la monnaie domestique de la fonction d'unité de compte. Avec la persistance d'une inflation élevée, les résidents sont poussés à effectuer la plupart de leurs transactions en devises. Ces dernières ne servent plus à financer la fuite des capitaux mais plutôt à réaliser les transactions à l'intérieur du pays, qu'il s'agisse de paiement sous forme manuelle ou des virements bancaires en devises. C'est à ce moment que la fonction de moyen de paiement est atteinte.

\subsubsection{La dollarisation par la pénurie des moyens de paiement}

En tant que contexte de la dollarisation, la pénurie de moyens de paiement peut être expliquée par l'hyperinflation et la déflation. La baisse de la valeur réelle de la masse monétaire provoque la pénurie des moyens de paiement. Cette baisse est, elle-même, le résultat d'un accroissement plus rapide du niveau général des prix des biens au-delà de l'émission monétaire. En conséquence, l'émission monétaire devient soumise à la hausse des prix et ne cesse de courir après les besoins. La valeur faciale des coupures s'effondre vite. Il faut très rapidement créer de nouveaux billets à valeur faciale plus élevée pour satisfaire les besoins de la circulation. L'effondrement de la valeur de ces nouveaux billets ne peut plus tarder. Il se crée alors un besoin extrême des moyens de paiement à valeur stable, une monnaie étrangère.

Avec la déflation, l'attentisme et une forte aversion au risque des agents se manifestent. Le retrait de la circulation d'une partie de la masse monétaire n'alimente 
plus correctement la masse monétaire. Mais au lieu de recourir directement à une monnaie étrangère, des moyens de paiement alternatifs interviennent (troc, monnaie marchandise,...)

\subsubsection{La dollarisation par la pénurie des biens}

La pénurie des biens, outre qu'elle se traduit par une hausse des prix, elle est source de manœuvre pour les vendeurs qui ne reçoivent en priorité que des demandeurs munis des devises. Par ailleurs, en plus d'être reçus en second lieu, les détenteurs de la monnaie nationale sont également victimes d'un prix majoré d'un certain pourcentage servant, aux yeux des vendeurs, de couverture de risque de change. Evincés, ces seconds demandeurs contribuent à l'accroissement de la demande des devises et de leur utilisation afin de bénéficier, eux aussi, d'une priorité et d'une prime sur ceux qui n'en disposeront pas encore. Ces derniers agissent de la sorte et le processus s'amplifie.

\subsubsection{La dollarisation par les réseaux transfrontaliers}

Le rayonnement régional d'une monnaie se traduit avant tout par sa circulation dans le strict voisinage de son pays d'origine. Mais, de façon générale, la force d'une monnaie conjuguée à la puissance commerciale de son pays sont les facteurs principaux qui poussent à son emploi dans une zone plus ou moins étendue à l'intérieur d'un pays voisin; c'est le cas du dollar des Etats-Unis qui d'abord utilisé tout au long de la frontière mexicaine dont certaines villes doivent leur dynamisme aux usines américaines qui y sont implantées, a par la suite eu un rôle puissant dans tout le Mexique (Vahavi, [2007], p.79). 


\subsubsection{La dollarisation par la pluralité monétaire légale}

Face à des crises économiques profonds ancrées dans des difficultés monétaires, des pays autorisent, favorisent et institutionnalisent l'usage déjà très répandu d'une monnaie étrangère. Il s'agit d'une officialisation d'une dollarisation de fait. Bien de situations illustrent une pareille dollarisation. A ce titre, dans un contexte de vingt ans de monter inexorable de l'inflation et deux années d'hyper-inflation et de chaos économique et politique, l'Argentine, au $1^{\text {er }}$ janvier 1991, a opté pour le système de «currencyboard» et de parité peso/dollar. Le dollar a été autorisé à circuler sous forme manuelle et la conversion du peso en dollar à parité a désormais été garantie. L'objectif de la réforme était de lier presque totalement l'émission monétaire aux réserves en devises, ce qui donnait du même coup au dollar une légitimité politique qu'il n'avait pas auparavant (Vahavi, [2007], pp. 79-80).

Cette analyse basée sur l'offre des monnaies montre que la dollarisation officielle n'est qu'une initiative souveraine et autonome. L'adoption d'une telle mesure est essentiellement justifiée par le désir de rétablir la stabilité des variables économiques : indice de prix, taux de change et d'intérêt, déficit budgétaire, balance de paiement, afin d'une insertion dans l'économie mondiale d'une élimination des distorsions monétaires et de change, d'une réactivation de la production et à la discipline budgétaire pour un développement national.

La dollarisation, traduisant, dans un monde globalisé, la force de la référence au dollar, peut paradoxalement induire, pour le pays qui adopte ce régime sans avoir une compétitivité suffisante, un biais antiexportation prononcé car sa capacité de pénétration des marchés étrangers s'effondre, en même temps que la monnaie forte - le dollar des Etats-Unis - incite aux 
importations faciles. Le pays s'expose ainsi, à terme, à se voir exclu de cette économie globalisée, alors qu'il croyait posséder la clef d'une insertion définitivement crédible en son sein.

\subsection{La dollarisation de l'économie en ville de Butembo 1.5.1. Les causes du recours au dollar à Butembo}

La dollarisation en ville de Butembo aurait des origines diverses. Des investigations menées à ce renseignent que la crise de substitution monétaire en faveur $\mathrm{du}$ dollar américain proviendrait d'une part au dysfonctionnement du système bancaire congolais et de la perméabilité des frontières du pays, surtout celle que la $R D C$ partage avec l'Ouganda. En effet, le dysfonctionnement du système bancaire congolais a bloqué le financement du commerce extérieur. Les opérations d'import-export ne se dénouaient plus facilement suite à la carence des devises et de la monnaie nationale dans la Banque Centrale ainsi que les banques agréées implantées à Butembo. (Tembo Paluku D., [2004], p.77).

Etant donné que le commerce connaissait la prospérité en ville de Butembo,les hommes d'affaires ont tiré avantage de la perméabilité de ses frontières. Ainsi donc, les autres pays africains, surtout les pays frontaliers de la $R D C$ où les opérateurs économiques écoulaient,d'ailleurs frauduleusement, des quantités importantes des matières précieuses constituent une source non négligeable. Ces exportations leur permettaient d'effectuer leurs paiements à l'étranger,d'où ils pouvaient revenir soit avec des marchandises, soit avec des devises étrangères - les dollars des Etats-Unis - qui, le plus souvent, alimentaient le marché parallèle de change. De plus,l'usage du dollar à Butembo résulte de son essor commercial 
considérable. De ce fait, une offre importante est née des transactions d'approvisionnement en produits manufacturés par les opérateurs économiques de certaines villes du pays.

Aussi, il sied de souligner que le trafic du «coltan » (Colombo Tantale) a attiré sur Butembo une masse considérable des dollars. En fait quelques opérateurs économiques du milieu achetaient ce minerai pour le compte des grandes firmes européennes. Il faut également noter le taux de change pratiqué en ville de Butembo,variant selon la volonté des plus nantis au détriment des plus démunis, était fixé par référence à celui pratiqué dans certaines grandes villes comme Goma, Kisangani, Kinshasa, Bukavu. Ayant constaté que le taux à Butembo était supérieur à celui des autres villes, plusieurs spéculateurs de ces villes sont venus arroser abondamment le marché local en devises américaines; et tout cela ne faisait qu'amplifier le phénomène.

\subsubsection{L'ampleur de l'usage du dollar dans l'économie de Butembo}

Butembo, une ville dont la vocation commerciale n'est plus à démontrer doit la survie de ses affaires à l'utilisation de la devise américaine. 93\% d'opérateurs économiques interrogés estiment que l'usage du dollar dans l'économie locale a été favorable à l'épanouissement de leur commerce. (Tembo Paluku D., [2004], pp.77-78)

En effet, une plus grande partie des ventes de biens et services s'y effectue en dollar. Les hommes d'affaires de Butembo libellent les prix des articles directement en dollar ou en monnaie nationale - le franc congolais -. Dans ce dernier cas, les prix sont régulièrement fixés avec un écart considérable susceptible de couvrir les risques de change du fait que la monnaie nationale vouée à l'échange est souvent fluctuante. Par cette pratique, les opérateurs économiques 
cherchent à conserver leurs marges bénéficiaires. Ainsi, les gagne-petit se considèrent défavorisés à convertir leurs avoirs des francs congolais en dollar auprès des cambistes. Dès lors, les consommateurs se trouvent dans l'obligation d'être en possession du dollar pour effectuer leurs achats et conserver le pouvoir d'achat pour les opérations ultérieures.

Ceux qui recourent aux micros crédits pour financer leurs petites activités commerciales sont probablement pénalisées par l'usage du dollar. Généralement, le crédit est octroyé et remboursé en dollar alors que les ventes des biens commercialisés s'effectuent en francs congolais. Cette opération présente ainsi des risques étant donné qu'il s'écoule un temps qui sépare le moment de vente de celui du remboursement du prêt. Au cours de cet intervalle de temps, toute éventuelle hausse du taux de change ronge la marge bénéficiaire pouvant même la faire disparaître et par conséquent occasionner des pertes dans l'activité de l'entrepreneur. Ce qui présente également un risque de non remboursement du prêt.

Présentement, en ville de Butembo, presque toutes les petites opérations s'effectuent en terme de dollars des Etats-Unis si bien qu'on serait tenté de dire que la monnaie nationale ne présente presque plus d'importance considérable. Ce phénomène constitue une dollarisation de fait de l'économie.

\section{ANALYSE ECONOMETRIQUE DE L'IMPACT DE LA DOLLARISATION SUR L'ECONOMIE EN VILLE DE BUTEMBO}

\subsection{Présentation des données}

Les données présentées dans cette section portent sur le taux de change du dollar des Etats Unis en franc Congolais, le taux de dollarisation et le taux d'inflation qui sont nos variables en étude. 
Tableau 1 Evolution du taux de change du dollar américain en franc congolais pratiqué sur le marché de Butembo de 2007-2011.

\begin{tabular}{|l|r|l|l|l|l|l|l|l|l|l|l|l|l|}
\hline A/M & J & F & M & A & M & J & J1 & A & S & O & N. & D. & M \\
\hline 2007 & 550 & 550 & 560 & 580 & 570 & 520 & 520 & 520 & 510 & 500 & 500 & 520 & 533,333 \\
\hline 2008 & 550 & 560 & 560 & 580 & 580 & 580 & 580 & 580 & 590 & 600 & 600 & 650 & 584,167 \\
\hline 2009 & 700 & 800 & 850 & 900 & 850 & 800 & 800 & 800 & 860 & 900 & 900 & 920 & 840 \\
\hline 2010 & 930 & 950 & 910 & 930 & 910 & 900 & 900 & 900 & 920 & 920 & 920 & 920 & 920,833 \\
\hline 2011 & 930 & 930 & 940 & 940 & 940 & 940 & 940 & 940 & 940 & 940 & 940 & 940 & 939,167 \\
\hline
\end{tabular}

$\mathrm{A} / \mathrm{M}=$ Annéé / Mois ; $\mathrm{M}=$ Moyenne

Source : Rapports annuels du bureau de l'économie nationale/ville de Butembo.

Le tableau présenté ci haut, nous donne l'évolution du taux de change de la devise américaine par rapport au franc congolais. Nous observons que le taux a généralement varié d'un mois à l'autre pendant notre période d'étude. Ce taux a globalement connu une augmentation, partant en moyenne de $530 \mathrm{Fc}$ à 2007 jusqu'à $940 \mathrm{Fc}$ l'année 2011 ; ce qui signifie qu'il a connu une hausse de $77,36 \%$ pour les cinq ans. La forte augmentation s'est observée entre les années 2008 et 2009 où le taux est passé moyennement de $580 \mathrm{Fc}$ à $840 \mathrm{Fc}$, soit une hausse de $44,83 \%$. En plus nous constatons une quasi stabilité du taux de change pendant les deux dernières années où le taux est resté dans l'intervalle de $910 \mathrm{Fc}$ à $950 \mathrm{Fc}$ pendant les 24 mois après une continuelle fluctuation à la hausse pour les années précédentes. Ce qui serait l'un des fruits de l'effort fourni par le gouvernement pour assurer une stabilité du cours de la monnaie nationale.

Dans le tableau qui suit nous présentons l'ampleur de l'intervention du dollar américain dans le circuit monétaire en ville de Butembo. 
Tableau 2 Estimation du taux de dollarisation en ville de Butembo de 2007-2011

\begin{tabular}{|r|r|l|l|r|}
\hline Année & $\begin{array}{l}\text { Valeur des } \\
\text { dépôts de Fc } \\
\text { en \$US }\end{array}$ & $\begin{array}{l}\text { Dépôts } \\
\text { en \$US }\end{array}$ & $\begin{array}{l}\text { Total des } \\
\text { dépôts }\end{array}$ & Taux en \% \\
\hline 2007 & 113321,94 & 1197647 & 1310968,94 & 533,36 \\
\hline 2008 & 118166,1 & 1608029 & 1726195,1 & 93,15 \\
\hline 2009 & 109025,66 & 1046087 & 2155112,66 & 94,94 \\
\hline 2010 & 112137,26 & 2364178 & 2576315,26 & 95,47 \\
\hline 2011 & 120982,05 & 1244546 & 2265528,05 & 94,66 \\
\hline
\end{tabular}

Source: Nos calculs effectués sur base des chiffres nous fournis par la COOPEC-COODEFI.

Les chiffres présentés dans le tableau ci-dessus dégagent un taux de dollarisation élevé en ville de Butembo pendant les cinq ans. Pour toute la période, le taux de dollarisation est estimé en plus de $90 \%$, cela manifeste une véritable défiance des habitants de Butembo à l'égard de la monnaie nationale; une crise de confiance qui est susceptible d'entamer la valeur du franc congolais du fait que cette monnaie ne remplirait plus efficacement les fonctions reconnues à une monnaie.

Concernant le taux d'inflation, celui-ci est calculé à partir des indices de prix à la consommation. Etant donné qu'il est très difficile d'inclure tous les produits de consommation dans le calcul des indices de prix, nous avons retenu quinze produits estimés d'être couramment consommés et/ou dont les prix sont plus sensibles. Ainsi avons-nous récolté les prix et les quantités-niveau d'approvisionnement de ces produits à partir des rapports annuels du Bureau Urbain de l'économie Nationale/Ville de Butembo.

En effet, ces données sur le prix et quantités sont mensuelles. $\mathrm{Du}$ fait de l'existence des mois d'essor économique mois exceptionnellement bons ou des mois de 
fluctuations saisonnières particulièrement mauvais-les données récoltées sont regroupées par trimestre. Ceci étant, notre période d'étude allant de 2007 à 2011 couvre 20 trimestres dont les chiffres présentés sont des moyennes arithmétiques des données mensuelles présentées en annexe.

En vue d'une présentation aisée des tableaux des prix et quantités, il nous a paru utile de symboliser les produits comme suit:X1= Cossettes de manioc, $\mathrm{X} 2=$ Haricots secs, X3 = Riz Local, X4= Pomme de terre, X5= Huile de palme, $\mathrm{X} 6=$ Poisson salé, $\mathrm{X} 7=$ Viande bovine, $\mathrm{X} 8=$ Savon Saibu, $\mathrm{X} 9=$ Essence Super, $\mathrm{X} 10=$ Bière Primus, $\mathrm{X} 11=$ Lait Nido, $\mathrm{X} 12=$ Sel de Cuisine, X13= Wax demisuper, X14= Lait de beauté Miss caroline, X15= Carte prépayée de communication/Airtel. Nous ne présentons pas les tableaux qui ont servi à l'élaboration des moyennes.

$\mathrm{Au}$ regard de ce qui précède, nous trouvons que certains produits manquent de chiffres du fait que le service de l'Economie Nationale n'en dispose pas (de X10 à X15); ensuite la destruction des archives de l'abattoir public a fait qu'il n'y ait pas de données sur la variable X7 (viande bovine) concernant notre période d'étude.

\subsection{Les outils d'analyse des données}

L'analyse de ces données présentées en annexe est effectuée par une approche économétrique. L'économétrie, branche de l'économie étant une application des méthodes statistiques et mathématiques à l'analyse des données économiques, va nous permettre de donner un contenu empirique et quantifié aux relations économiques en étude. Pour cette étude, le calcul des indices de prix nous permet de bien saisir la variation des prix et donc le taux d'inflation et pour mesurer les différentes liaisons entre les variables taux de dollarisation, taux de change et taux d'inflation, 
nous faisons usage des techniques de la corrélation et régression simples. (Pigagniol B., [1978], p.22)

\subsection{1.. Les Indices de prix à la Consommation}

L'indice est un nombre exprimant un rapport entre deux grandeurs. Il permet de caractériser par un nombre unique la variation d'une variable ou d'un ensemble de variables entre deux périodes. Dans cette étude, nous usons de la formule dite de Laspeyres (1)-pondération à 1'aide de 1'époque de base et celle dite de Paasche (2) pondération à l'aide de l'époque courante. Les inconvénients de l'une et l'autre formule se dissoudront par l'application, sur les deux précédentes, de la formule dite d'Irving Fisher (3) qui est une moyenne géométrique des indices de Laspeyres et de Paasche. Ces indices sont donnés par les formules suivantes :

$$
\begin{aligned}
& L_{t / 1(p)}=100 \frac{\sum_{i=1}^{n} P_{i, t} \times Q_{i, 1}}{\sum_{i=1}^{n} P_{i, 1} \times Q_{i, 1}}(1), \\
& L_{t / 1(p)}=100 \frac{\sum_{i=1}^{n} P_{i, t} \times Q_{i, t}}{\sum_{i=1}^{n} P_{i, 1} \times Q_{i, t}} \\
& \text { et } F_{t / 1(P)}=\sqrt{L \times P}
\end{aligned}
$$

où $\mathrm{L}$ est l'indice de Laspeyres et $\mathrm{P}$ est celui de Paasche. C'est ce dernier indice dont nous userons pour dégager les indices annuels et par conséquent le taux d'inflation. Dans (1) et (2) $t$ représente l'année courante ou période courante ; $l$ l'année de base ou période de base, $P_{i, t}$ le prix i à la période $t ; Q_{i, 1}$, la quantité $i$ à la période $l ; P_{i l}$, le prix i à la période $l, n$ le nombre de périodes et $P$ indique qu'il s'agit de 1 'indice de prix. Mais aussi $t ; 1 ; P_{i t}: q_{i, 1} ; P_{i l} ; n$ et $(p)$ 
signifient la même chose que précédemment et $q_{i, t}$ désigne la quantité $i$ à la période $t$.

\subsubsection{La régression et la corrélation simples}

$\mathrm{La}$ régression est un ajustement des séries statistiques par des droites, la corrélation étant supposée linéaire. Elle permet d'obtenir une représentation quantitative simplifiée, mais approximative, des liaisons existant entre plusieurs variables. La droite de régression de $\mathrm{y}$ en $\mathrm{x}-Y_{i}=a x_{1}+b$-permet de calculer une valeur

numérique approximative $Y$ pour chaque valeur numérique observée de la série $\mathrm{x}$.

Dans l'explication du modèle, interviennent d'autres variables aléatoires inconnues aussi explicatives que $\mathrm{x}$ mais non explicitées. Les effets de ces facteurs sont représentés par $\varepsilon_{i}$. Le modèle s'écrit alors $Y_{i}=a x i+b+\varepsilon_{i}$

L'estimation des paramètres $a$ et $b$ du modèle est faite par la méthode de moindres carrés ordinaires (MCO) minimisant $\varepsilon_{i}$ et par laquelle $a$ est estimé par $a$ et b par $b$. Ainsi, le modèle estimé devient $Y_{i}=\hat{a} x_{i}+b$ où $a$ est la pente de droite et $b$ le terme constant. La corrélation mesure le degré de liaison existant entre les phénomènes représentés par des variables en régression. Cette liaison est évaluée par le coefficient de corrélation $r$ calculé par la formule suivante :

$$
r_{x y}=\frac{\sum_{i=1}^{n}(x i-\bar{x})(y i-\bar{y})}{\sqrt{\sum_{i=1}^{n}(x i-\bar{x})^{2} \times \sum_{i=1}^{n}\left(Y_{i}-\bar{Y}\right)^{2}}} .
$$




\subsection{Mesure du taux d'inflation en ville de Butembo}

Comme signifié précédemment, la mesure du taux d'inflation est effectuée en passant par le calcul des indices de prix à la consommation.

Tableau 3Indices trimestriels de prix à la consommation

\begin{tabular}{|c|c|c|c|}
\hline Trimestres/Indices & $L t / 1(p)$ & $\mathbf{P t} / \mathbf{1}(\mathbf{P})$ & $\mathrm{Ft} / \mathbf{1}(\mathrm{P})$ \\
\hline 1 & 100 & 100 & 100 \\
\hline 2 & 108,196694 & 110,375402 & 109,281 \\
\hline 3 & 134,626131 & 144,335402 & 139,396 \\
\hline 4 & 142,176506 & 147,746761 & 144,935 \\
\hline 5 & 154,035404 & 161,767132 & 157,854 \\
\hline 6 & 178,217839 & 187,150232 & 182,629 \\
\hline 7 & 194,089375 & 223,13939 & 208,108 \\
\hline 8 & 197,894905 & 221,764667 & 209,49 \\
\hline 9 & 236,761281 & 252,953082 & 244,723 \\
\hline 10 & 379,250371 & 414,735329 & 396,596 \\
\hline 11 & 330,524763 & 336,214269 & 333,357 \\
\hline 12 & 385,25694 & 379,783223 & 382,51 \\
\hline 13 & 390,015245 & 382,272075 & 386,124 \\
\hline 14 & 379,678181 & 373,007095 & 376,328 \\
\hline 15 & 348,819119 & 315,005531 & 331,481 \\
\hline 16 & 364,027953 & 355,064136 & 359,518 \\
\hline 17 & 411,249482 & 403,054941 & 407,132 \\
\hline 18 & 433,872802 & 503,773092 & 467,518 \\
\hline 19 & 452,857009 & 440,302695 & 446,536 \\
\hline 20 & 491,352145 & 481,309761 & 486,305 \\
\hline
\end{tabular}

Source : Nos calculs sur base des données des tableaux 3 et 4

Comme vu précédemment dans les formules, le calcul des indices de prix pondèrent les prix par les quantités.

Au regard de ce tableau, ne considérant que l'indice de Ficher $F_{t / 1(P)}$, nous constatons que la variation des prix a 
été croissante au cours de notre période d'étude. De manière globale, du premier au vingtième trimestre, les prix ont atteint $486,305 \%$. Ceci signifie que les prix ont été pour l'ensemble de la période 4,86305 fois ceux du premier trimestre 2007, c'est-à-dire que les prix ont augmenté de $386,3 \%$ par rapport au premier trimestre 2007.

Ainsi exposés les indices trimestriels, il convient de calculer maintenant les indices annuels pour en déduire le taux d'inflation annuel constituant l'une de nos variables en étude.

Cependant, le problème de confrontation de ces indices annuels avec les indices trimestriels est très délicat. Pour ce fait, on procède au calcul de l'indice annuel en calculant la moyenne des indices pour quatre trimestres de chaque année, en la divisant par la moyenne des indices de l'année de base puis en la multipliant par cent. Ceci nous conduit au tableau ci-dessous :

Tableau 4 : Indices annuels des prix à la consommation en

\begin{tabular}{|l|c|c|c|c|c|}
\hline Années & 2007 & 2008 & 2009 & 2010 & 2011 \\
\hline Indices & 100 & 153,578 & 274,95 & 294,452 & 366,177 \\
\hline
\end{tabular}

Source : Nos calculs sur base du tableau 7 en annexe

Comme observé déjà au niveau du calcul des indices trimestriels, nous trouvons que les indices annuels des prix ont évolué de manière croissante pendant les cinq ans d'étude. Cela signifie que les prix des prix des produits ont connu une hausse d'une année à l'autre sur le marché de Butembo. De façon globale, les prix ont atteint $366,177 \%$ de 2007 à 2011 ; ce qui signifie qu'en 2011 les prix ont été 3,66177 fois ceux de l'année 2007, c'est-à-dire une augmentation de $266,1 \%$ par rapport au premier trimestre 2007. 
À partir de ces indices moyens annuels des prix à la consommation, calculons le taux d'inflation en ville de Butembo en application de la formule suivante :

Taux d'inf lation $=\frac{I_{t}-T_{0}}{I_{0}} \times 100$ Avec $I_{t}=$ Indice de prix à la période $t, I_{0}=$ Indice de prix à la période précédente. D'où, le tableau ci-dessous.

\section{Tableau 5 : Evolution du taux d'inflation en ville de}

Butembo de 2007 à 2011 en \%

\begin{tabular}{|l|c|c|c|c|c|}
\hline Années & 2007 & 2008 & 2009 & 2010 & 2011 \\
\hline Indices & 1 & 53,578 & 79,03 & 7,093 & 24,359 \\
\hline
\end{tabular}

Source : Nos calculs sur base du tableau 4

Partant de l'année de référence (2007), il s'est observé une hausse du taux d'inflation pendant les années 2008 et 2009 respectivement de $53,578 \%$ et $79,03 \%$. De 2009 à 2010 , ce taux a chuté de $79,03 \%$ à $7,093 \%$. Enfin, de 2010 à 2011 , ce taux est passé de $7,093 \%$ à $24,359 \%$. De ce qui précède, nous constatons que le taux d'inflation a été faible pour les deux dernières années comparativement aux années précédentes. Cela serait l'un des effets de la stabilité monétaire observée ces mêmes années. Ainsi donc, nous passons à l'étude des liaisons entre nos différentes variables dans les sections suivantes.

\subsection{Régression entre taux de dollarisation et taux de change}

\subsubsection{Expression mathématique du phénomène}

La relation entre le taux de dollarisation et le taux de change en ville de Butembo est exprimée à travers la figure qui suit : 
Graphique 1: Relation entre taux de dollarisation et taux de change.

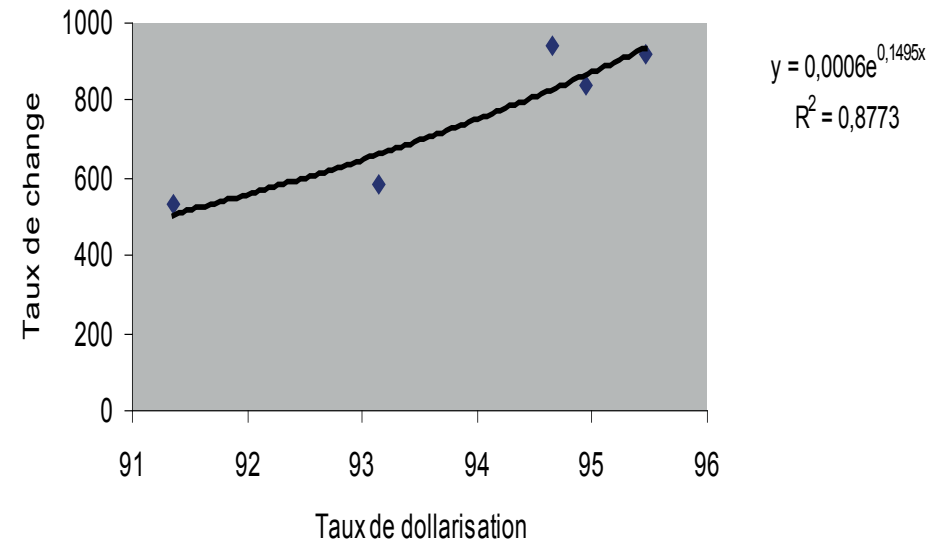

Il s'observe une liaison croissante de forme exponentielle entre le taux de dollarisation et le taux de change comme le montre la courbe de tendance $Y=0,0006 e^{0,1495 x}$. Le taux de dollarisation influe sur le taux de change en $87,73 \%$.

\subsubsection{Corrélation entre taux de dollarisation et taux de change.}

Pour bien vérifier l'existence d'une corrélation entre ces deux variables en étude, nous procédons par le test du coefficient de corrélation. Ses étapes sont les suivantes :

1. Hypothèse : $-\rho=0$; pas de corrélation entre les variables - $\rho \neq 0$; il y a une corrélation entre les variables.

2. $\propto=5 \% ; n=5 \prec 30$ nous utilisons le test $\mathrm{T}$ de Student 
3. Règle de décision; Rejeter Ho si $\left|T_{c a l}\right| \succ T_{\text {th }}$ avec $T_{t h ; n-k}=T_{0,025 ; 3}=3,182$

\section{Calcul :}

$$
T_{c a l}=\frac{R \cdot \sqrt{n-2}}{\sqrt{1-R^{2}}}=\frac{0,936642941 \cdot \sqrt{3}}{\sqrt{1-0,8773}}=4,631401273
$$

\section{5- Décision}

Nous constatons que :

$$
\left|T_{\text {cal }}\right|=4,632 \succ T_{\text {th }}=3,182 \text {. }
$$

Nous rejetons Ho au seuil de signification de 5\% et concluons qu'il existe une corrélation entre le taux de dollarisation et le taux de change. Le coefficient de corrélation $\mathrm{R}=0,936642941$, proche de 1 ; il y a donc une forte liaison entre ces variables. Cela signifie qu'au fur et à mesure que les acteurs économiques de Butembo portent leur intérêt à la devise américaine, il s'observe une dépréciation de la monnaie nationale s'expliquant par la hausse du taux de change du dollar américain en Franc Congolais. A la lumière de ces résultats, notre première hypothèse est bel et bien confirmée.

\subsubsection{Régression entre taux de dollarisation et taux d'inflation}

\section{a. Expression mathématique du phénomène}

La liaison entre les variables taux de dollarisation et taux d'inflation est visualisée par la graphique ci-dessous : 
Graphique 2: Relation entre taux de dollarisation et taux d'inflation

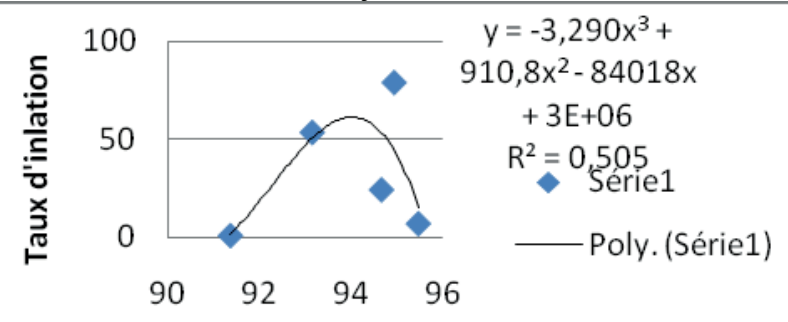

Taux de dollarisation

Au regard de cette figure, nous nous rendons compte d'une liaison entre le taux de dollarisation et le taux d'inflation mieux ajustée par le modèle polynomiale du troisième degré de courbe de tendance $Y=-3,2906 X^{3}+910,88 X^{2}-84018 X+3 E+06$.

Le coefficient de détermination $R^{2}=0,5057$ exprime une influence du taux de dollarisation au taux d'inflation en $50,57 \%$; une liaison pas très déterminante.

\section{b. Tests Statistiques du modèle}

Le modèle polynomiale ci-dessus est un modèle à trois variables explicatives qui nécessite une taille d'observations élevée pour l'effectivité des tests du modèle. Cependant, la taille d'observations de notre analyse est minime ; ce qui constitue une difficulté à la détermination de la signification globale du modèle ainsi que de paramètres de régression. Dans le présent modèle, le coefficient de corrélation $\mathrm{R}=0,711125867$ témoigne de la liaison entre les variables en étude. Il convient donc de retenir l'hypothèse que le taux d'inflation est expliqué en $50,57 \%$ par le taux de dollarisation. Cela veut dire que les 
mécanismes de variations des prix des produits sur le marché de Butembo tiennent à un certain seuil sur l'usage de l'une ou de l'autre monnaie.

De ce fait, il y aurait d'autres facteurs qui influencent la variation du niveau général des prix en ville de Butembo tel que l'augmentation de la demande non adaptée à l'offre, les tendances spéculatives des opérateurs économiques, une éventuelle hausse du prix du carburant influençant directement les prix des autres biens, la mémoire inflationniste de la population, la hausse du taux de change et tant d'autres.

Il sied d'analyser une éventuelle liaison entre le taux de change et le taux d'inflation.

2.5. Régression entre taux de change et taux d'inflation.

2.5.1. Expression mathématique du phénomène.

La relation entre ces deux variables est exprimée par la représentation graphique ci-dessous :

Graphique 3: Liaison entre le taux de change et le taux d'inflation

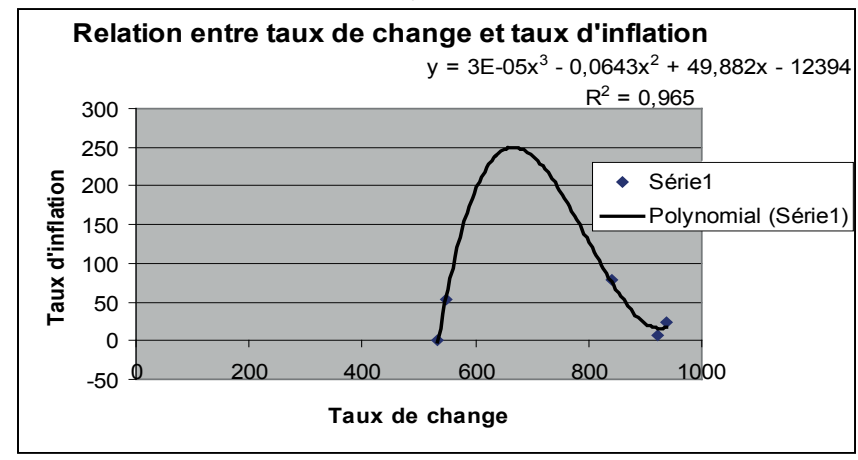

Il y a une relation entre le taux de change et le taux d'inflation ajustée également par le modèle polynomiale de 
troisième degré de courbe d'équation ${ }^{29}$ : $Y=3 E-05 X^{3}-0,0643 X^{2}+49,882 X-12394$.

Le coefficient de détermination $\mathrm{R}^{2}=0,965$ indique que le taux de change influence le taux d'inflation en $96,5 \%$.

\subsubsection{Tests Statistiques du modèle}

L'observation y relative faite à la section précédente est aussi vérifiable ici. Dans ce modèle, le coefficient de corrélation $\mathrm{R}=0,982344135$, proche de 1 , témoigne de l'existence d'une forte liaison entre les variables en étude. Néanmoins, il convient tout de même de confirmer cette affirmation par un test de coefficient de corrélation effectué ici-bas.

\subsubsection{Corrélation entre taux de change et taux d'inflation}

L'application dudit test suit la procédure suivante :

1. Hypothèses : - Ho : $\rho=0$; pas de corrélation entre les variables

- Ha : $\rho \neq 0$;il y a une corrélation entre les variables

2. $\propto=5 \% ; n=5 \prec 30$, nous utilisons le test $\mathrm{T}$ de Student

3. Règle de décision; Rejeter Ho si $T_{C a l} \succ T_{t h}=3,182$

\section{Calcul :}

$$
T_{c a l}=\frac{R \cdot \sqrt{n-2}}{\sqrt{1-R^{2}}}=\frac{0,982344135 \cdot \sqrt{5-2}}{\sqrt{1-0,965}}=9,094739451
$$

\section{Décision}

Nous trouvons que $T_{\text {Cal }} \succ T_{T h}$, nous rejetons l'hypothèse nulle au seuil de $5 \%$ et concluons qu'il y a une corrélation entre le taux de change et le taux d'inflation.

${ }^{29}$ Les Modèles curvilignes n'admettant pas des tests de régression, sauf linéarisation pour les modèles exponentiels. 
Ainsi, le taux de change influence le niveau de prix sur le marché de Butembo. Le coefficient de détermination $\mathrm{R}^{2}=0,965$ a signifié que la variation du niveau de prix est expliquée en $96,5 \%$ par le taux de change qui est à son tour expliqué par le taux de dollarisation. Au regard des résultats fournis par ces deux modèles polynomiales, nous trouvons que l'explication du taux d'inflation par le taux de dollarisation est plus signifiée par la relation: le taux de dollarisation influence le taux de change quand le taux de change influence le taux d'inflation; par conséquent, le taux de dollarisation exerce une certaine influence sur le taux d'inflation. Cette influence n'est pas trop déterminante car n'étant que de $50,57 \%$; ce qui nous permet de nuancer notre seconde hypothèse.

\section{CONCLUSION, LIMITES ET FUTURES VOIES DE RECHERCHE}

La présente étude portant sur l' "Impact de la dollarisation sur les activités économiques en ville de Butembo » tournait autour d'une série de questions qui ont constitué notre problématique.

En effet, ce travail a eu comme préoccupation de mesurer les effets de la dollarisation sur l'état d'appréciation de la monnaie nationale, le franc congolais et sur le niveau de la variation des prix à la consommation des produits en ville de Butembo. En guise d'hypothèses vérifiables, nous avons estimé que la dollarisation provoquerait une dépréciation de la monnaie nationale face au dollar américain sur le marché de Butembo et qu'elle y influencerait le niveau général des prix des produits.

S'agissant de l'impact de la dollarisation sur l'état d'appréciation du franc congolais, nos analyses ont révélé une liaison croissante de forme exponentielle symbolisée 
par la courbe $Y=0,0006 e^{0,1495 x}$ entre le taux de dollarisation et le taux de change du dollar américain -des Etats Unis- en franc congolais. Après linéarisation du modèle et tests du modèle, l'analyse corrélative a conclu qu'il existe une corrélation entre les deux variables, le coefficient de détermination $\mathrm{R} 2=0,8773$ indique que le taux de dollarisation influe sur le taux de change en $87,73 \%$. Cela signifie que l'augmentation du taux de dollarisation provoque une dépréciation du franc congolais se traduisant par la hausse du taux de change du dollar en francs congolais. Cela nous a permis de confirmer notre première hypothèse selon laquelle la dollarisation provoquerait une dépréciation de la monnaie nationale face au dollar américain sur le marché de Butembo.

Concernant l'influence de cette dollarisation sur le taux d'inflation, nos analyses ont fait ressortir une liaison de modèle polynomiale entre ces variables de courbe $\mathrm{Y}=-3,2906 \mathrm{X} 3+910,88 \mathrm{X} 2-84018 \mathrm{X}+3 \mathrm{E}+06$ avec un coefficient de détermination $\mathrm{R} 2=0,5057$ indiquant une influence plus ou moins significative du taux de dollarisation sur le taux d'inflation; cette influence étant de $50,57 \%$.

De plus, l'analyse de la relation entre le taux de change et le taux d'inflation a signifié l'existence d'une relation de modèle polynomiale entre ces deux variables de courbe $\quad \mathrm{Y}=3 \mathrm{E}-05 \mathrm{X} 3-0.0643 \mathrm{X} 2+49.882 \mathrm{X}-12394$. Le coefficient de détermination $\mathrm{R}^{2}=0,965$ signifie que le taux de change explique le taux d'inflation en 96.5\%. Au regard des résultats de ces deux modèles, nous constatons que l'explication du taux d'inflation par le taux de dollarisation est mieux signifiée par la relation : "le taux de dollarisation influence le taux de change, lequel taux de change explique le taux d'inflation ; Par conséquent, le taux de dollarisation 
exerce une certaine influence sur le niveau des prix', Le degré d'explication du niveau de prix par le taux de dollarisation n'étant que de $50,57 \%$ indique que cette influence n'est pas tellement grande. Ceci nous permet de nuancer notre seconde hypothèse stipulant que la dollarisation influencerait le niveau général des prix des produits sur le marché local, relation dans laquelle, la variation du taux de change constituerait un effet de transition.

De tout ce qui précède, nous comprenons que la tendance de faire recours à la devise américaine entame la valeur de la devise nationale et joue sur le niveau des prix des produits en ville de Butembo. De ce fait, à côté des efforts fournis par le gouvernement pour la stabilisation économique observée, il convient tout de même de penser à une éducation régulière de la population sur différents mécanismes économiques afin qu'elle ne dérègle pas de sa propre volonté les conditions du marché. En plus, le gouvernement a intérêt à bien définir sa politique budgétaire en vue de stimuler la croissance économique et ainsi maintenir forte l'économie nationale.

Néanmoins, nous supposons avoir lancé une base de réflexion proportionnelle à nos capacités. Ainsi pensonsnous que les recherches à venir s'en serviront pour d'autres analyses dans ce vaste champ d'investigation, tel que l'étude d'indexation du prix de vente des produits manufacturés sur le marché de Butembo.

Au-delà de l'intérêt de ces résultats, il faudra reconnaître plusieurs limites à cette étude. Tout d'abord comme dans toute étude des faits économiques, il se pose un problème de quantification parfaite des observations partant du fait que la plupart des statistiques officielles en $R D C$ sont quasi-absentes; tandis que l'aspect évolutive est beaucoup plus suppositoire en prétendant que le temps peut 
rester inchangé. Néanmoins, les résultats de nos investigations ne sont pas contraires à ceux de nos prédécesseurs.

\section{REFERENCES BIBLIOGRAPHIQUES}

H. Bourguinat, \& L. Dohni (Juillet 2002),

«La Dollarisation comme solution en dernier ressort», in Revue française d'économie, vol. XVI, juillet 2002

Calvo, G. A.and C. A. Vegh(1993),

"Exchange Rates Based Stabilization under Imperfect Credibility", In: Frisch, H., Worgotter, A. (Eds.), OpenEconomy Macroeconomics, McMillan, London

CAPUL, J. Y. et GARNIER, O.

(1996),Dictionnaire d'économie et des sciences sociales, éd Hatier, Paris, 527p.

GAETANO A et KEISTER T,

«Dollarisation: que nous enseigne la théorie?» in Problème économiques $\mathrm{N}^{\circ} 2748$, p13-18.

KAMBALE SEKERAVITI ;

Des défis et contraintes de la politique monétaire congolaise: une approche macroéconomique, Mémoire, UCG, 2007-2008

MONIQUE BEZIADE ;

PIGAGNIOL B.,

La monnaie, $2^{e}$ édition Masson, Paris, 1985, 461p

TEMBO PALUKU D ;

Statistique et Econométrie, éd. Dalloz, Paris, 1978,180p

«La problématique de stabilisation du taux de change en ville de Butembo » in Parcours et initiatives, Vol II N², UCG, p75-85

VAHAVI MULUME B ;

Instabilité Sociopolitique et substitution des monnaie: Essai sur la dollarisation en République Démocratique du Congo, Thèse de doctorat, Université PARIS XII VAL DE MARNE, 470p. 